\title{
Evaluation of DC-AC Single-Phase Solid-State Transformers
}

\author{
J. Bosso, Member, IEEE, M. Llomplat, G. Oggier, Member, IEEE, and G. García, Senior Member, IEEE
}

\begin{abstract}
This work presents an evaluation of three topologies of DC-AC single-phase solid-state transformers, for applications in electric microgrids. The main features of these topologies are galvanic isolation, capability to control the power flow bidirectionally, soft switching and unit power factor under open loop control. The principle of operation of each topology is described and the modulation strategy used is detailed. A comparison of the THDi of the current waveform injected into the grid, the power factor and the utilization factor of the semiconductor switches for each topology is presented. As a result, the most suitable topology for the integration of microgrids with high penetration of renewable energies to the grid is determined. Simulation results are presented to validate the theoretical analysis.
\end{abstract}

Index Terms - Isolated DC-AC converter, Unity power factor, Phase-shift modulation, Pulse-width modulation, Soft switching.

\section{INTRODUCCIÓN}

$\mathrm{U}$ NA microrred es un sistema de distribución de corriente alterna (CA), corriente continua (CC) o $\mathrm{CC}$ en combinación con $\mathrm{CA}$, junto con fuentes de generación distribuida, dispositivos de almacenamiento y cargas en combinación con convertidores electrónicos de potencia.

$\mathrm{Si}$ se considera que los generadores eólicos operan a frecuencia variable, los paneles fotovoltaicos generan energía en CC y la mayoría de los sistemas almacenadores de energía son de CC [1][2], resulta conveniente pensar en una integración de todas estas fuentes de energías en una barra de $\mathrm{CC}$ en conjunto con convertidores electrónicos, para controlar el flujo de energía en la misma [3][4]. A su vez, esta microrred puede encontrarse aislada o interconectada con el Sistema Eléctrico de Potencia (SEP) a través de una interfaz electrónica bidireccional que controle eficientemente el flujo de energía desde y/o hacia la microrred [5]. Esta interconexión puede poseer aislación galvánica, con objetivos de protección y facilitar la adaptación de los diferentes niveles de tensión.

J. E. Bosso, Universidad Nacional de Río Cuarto y CONICET, Córdoba, Argentina,jbosso@ieee.org.

M. Llomplat, Universidad Nacional de Río Cuarto y CONICET, Córdoba, Argentina, marcelollomplat@gmail.com.

G. G. Oggier, Universidad Nacional de Río Cuarto y CONICET, Córdoba, Argentina,goggier@ieee.org.

G. O. García, Universidad Nacional de Río Cuarto y CONICET, Córdoba, Argentina, g.garcia@ieee.org.
En la Fig. 1(a) se muestra el esquema de una microrred de $\mathrm{CC}$ interconectada con el sistema eléctrico de potencia a través de una interfaz electrónica CA-CC bidireccional.

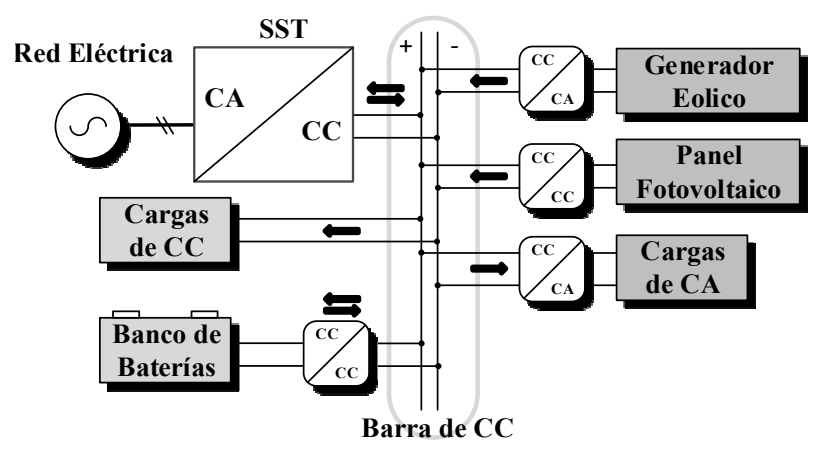

(a)

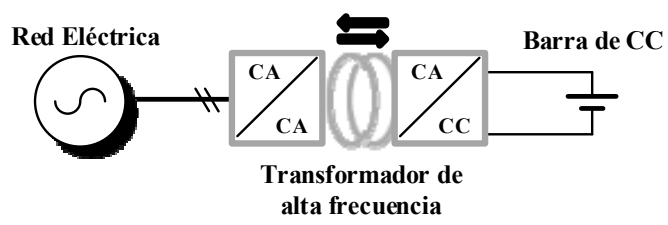

(b)

Fig. 1. (a) Esquema de microrred de CC interconectada con el SEP. (b) Configuración de convertidor CA-CC aislado.

Los convertidores electrónicos de potencia utilizados para interconectar redes eléctricas de diferentes características son comúnmente denominados transformadores de estado sólido (Solid-State Transformer, SST). Estos convertidores poseen aislación galvánica mediante la utilización de transformadoresde alta frecuencia, permiten controlar el flujo de energía de manera bidireccional independientemente de variaciones en las características eléctricas de la red [6]-[8], por lo cual son reductores/elevadores de tensión, y en conjunto con las tecnologías de la información y comunicación (TIC), los SST facilitan la planificación, operación y protección del SEP [9].

Se han encontrado en la bibliografía topologías de SST monofásicos, los cuales realizan la conversión CC-CA de manera aislada aplicando un determinado desfase entre las tensiones a bornes del transformador en función de la potencia requerida. Estas topologías realizan la conversión CC-CA bidireccional y aislada mediante la utilización de un convertidor de CA de baja frecuencia a CA de alta frecuencia, un transformador de alta frecuencia y un convertidor de CA de alta frecuencia a $\mathrm{CC}$, como se muestra en la configuración de 
la Fig. 1(b), sin la necesidad de una etapa de CC intermedia [10]-[13], prescindiendo de la utilización de capacitores y de esta manera lograr incrementar la densidad de potencia y disminuir los costos de mantenimiento del convertidor. Las topologías mencionadas funcionan con el mismo principio de operación de los convertidores CC-CC con puentes duales activos (CPDA), en los cuales la potencia transferida se regula aplicando un determinado desfase entre las tensiones a bornes del transformador (phase shift modulation, PSM) [14]. A continuación, se presenta un resumen del estado del arte de las estrategias de modulación que pueden aplicarse a este tipo de convertidores.

En el trabajo presentado en [15] se analizan las pérdidas de un CPDA cuando se opera con cargas reducidas y amplia variación de las tensiones de entrada y de salida, cuando el convertidor se controla por medio de una modulación por ancho de pulso (PWM) junto con la modulación PSM. Como resultado, se logran disminuir las corrientes pico y una reducción significativa en el tamaño del transformador.

En [16] se presenta una estrategia de optimización para minimizar las corrientes en los inductores y en los transformadores, y en consecuencia, las pérdidas de conducción. La propuesta de modular la tensión generada por uno solo de los puentes (one/single H-Bridge modulation, SHBM) fue introducido en [17] y ampliado en [18], donde se implementa un control que maximiza el rendimiento del convertidor mediante la minimización de las pérdidas en la llaves semiconductoras y en el transformador.

Un caso particular de la SHBM, denominada como operación en modo interno, fue presentada en [19] para topologías de dos y tres puertos, la cual consiste en modular la tensión generada por uno de los puentes y el desfase de las tensiones generadas por ambos puentes para conseguir que en uno de los puentes las llaves semiconductoras conmuten con corriente cero (zero current switching, ZCS) y conmutar con tensión cero (zero voltage switching, ZVS) a las llaves semiconductoras del otro puente.

En [20] se presentó una estrategia de modulación aplicada a un convertidor CC-CA trifásico que permite reducir las pérdidas de conmutación en las llaves semiconductoras del convertidor, permitiendo que las llaves del lado de CA conmuten con ZCS y las del lado de CC con ZVS.

En este trabajo, se selecciona la estrategia de modulación presentada en [20], aplicada a las topologías monofásicas que se analizan en las siguientes secciones.

Este artículo presenta una evaluación de tres topologías de SST CC-CA monofásicas, aisladas, bidireccionales, elevadoras y reductoras de tensión que operan con factor de potencia unitario a lazo abierto de control. Se describe el principio de funcionamiento de cada topología y se detalla la estrategia de modulación utilizada. Luego se presenta una comparación de sus características principales a través de los resultados de la simulación, lo que permite determinar la topología más adecuada para estas aplicaciones.

El presente trabajo está organizado de la siguiente manera: en la sección II se describen las topologías propuestas y se explica el principio de funcionamiento. En la sección III se describe la estrategia de modulación. En la sección IV se presentan resultados de simulación para evaluar y comparar el desempeño de cada topología. Finalmente, en la sección V, se presentan las conclusiones.

\section{DESCRIPCIÓN DE LAS TOPOLOGÍAS Y PRINCIPIO DE FUNCIONAMIENTO}

En esta sección se presentan tres topologías de SST utilizadas para interconectar una red monofásica de CA convencional, con una barra de CC. Estas topologías tienen como característica común de ser elevadoras y reductoras de tensión, permiten controlar el flujo de energía de manera bidireccional y son aisladas por medio de transformadores de alta frecuencia.

En este trabajo se considera que la tensión de la barra de $\mathrm{CC}, V_{c c}$, es constante en un período conmutación y que la tensión de la red de CA monofásica puede definirse por la siguiente ecuación,

$$
v_{c a}=V_{c a} \sin \left(\omega_{r} t\right)
$$

donde $V_{c a}$ es el valor pico de la tensión de fase, $\omega_{r}=2 \pi f_{r}$, y $f_{r}$ es la frecuencia de la red.

\section{A. Topología $A$}

En la Fig. 2 se muestra un esquema del convertidor presentado en [10], definido en este trabajo como Topología A. Este convertidor está compuesto por dos puentes completos, formado por dos piernas de semiconductores cada uno, uno utilizado como interfaz del lado de CC y el otro como interfaz del lado de CA. El puente del lado de CC se implementa con llaves unidireccionales con sus correspondientes diodos en antiparalelo y es el encargado de imponer la tensión modulada $v_{S e c}$ entre los bornes $b$ y $b$, que representan los puntos medios de cada pierna del puente de CC. El puente que se conecta del lado de CA, se implementa con llaves bidireccionales en tensión y en corriente y es el encargado de imponer la tensión modulada $v_{\text {Prim }}$ entre los bornes $a$ y $a^{\prime}$ del lado de CA del transformador de alta frecuencia, $\operatorname{Tr} A$.

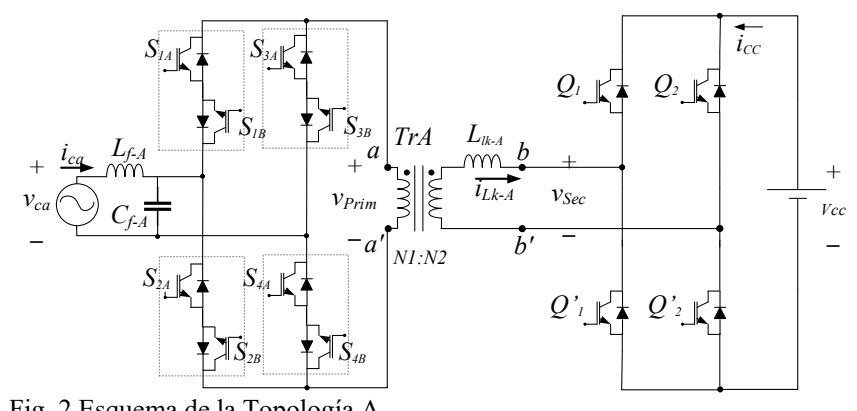

Fig. 2.Esquema de la Topología A.

Un transformador de alta frecuencia, junto con una inductancia auxiliar $L_{l k}$, interconecta ambos puentes. La relación de transformación se define como $\mathrm{n}=N 2 / N 1, N 1$ representa el número de espiras del lado de $\mathrm{CA}$ y $N 2$ representa el número de espiras del lado de CC.

Con el fin de eliminar las componentes armónicas en la corriente de la red de CA, $i_{c a}$, provocadas por la conmutación de las llaves semiconductoras, es necesario utilizar un filtro 
pasa bajos entre la red eléctrica y el convertidor de potencia, como se representa en la Fig. 2 [11].

\section{B. Topología $B$}

En la Fig. 3 se muestra un esquema del convertidor presentado en [12], definido en este trabajo como Topología B. El puente del lado de CA se implementa por dos llaves bidireccionales en tensión y en corriente en configuración semipuente, las cuales se conectan a un transformador monofásico de alta frecuencia, $\operatorname{Tr} B$, cuyo punto medio se conecta al neutro de la red de CA. Este puente es el encargado de imponer la tensión modulada $v_{\text {Prim }}$ entre los bornes $a$ y am, y entre $a m$ y $a$, respectivamente.

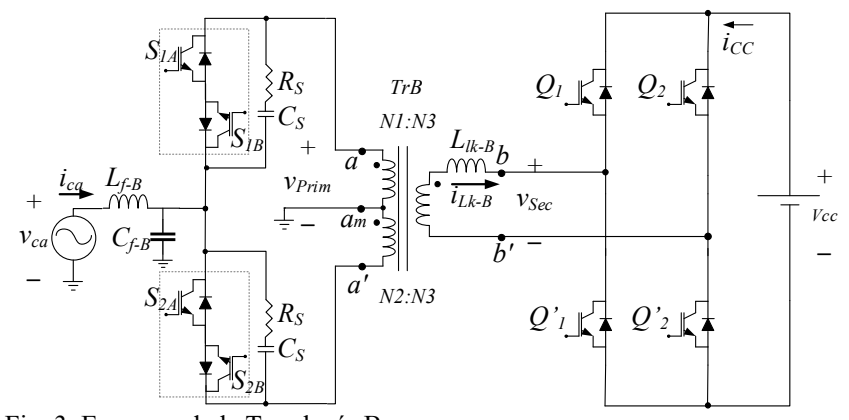

Fig. 3. Esquema de la Topología B.

En esta topología pueden producirse sobretensiones a bornes de las llaves semiconductoras del lado de CA, durante el apagado de las mismas, debido a la energía almacenada en la inductancia de dispersión del transformador, siendo necesario agregar redes amortiguadoras o snubbers en paralelo con las llaves [20]. En la Fig. 3, estas redes se representan por un capacitor, $C_{\mathrm{S}}$, y una resistencia, $R_{\mathrm{S}}$, en serie.

El puente del lado de CC se implementa de manera similar a la topología $\mathrm{A}$. La relación de transformación se define como $n=\mathrm{N} 3 /(\mathrm{N} 1+\mathrm{N} 2)$ siendo $\mathrm{N} 1=\mathrm{N} 2, \mathrm{~N} 1$ y N2 representan el número de espiras del lado de $\mathrm{CA}$ y $\mathrm{N} 3$ representa el número de espiras del lado de CC.

\section{Topología $C$}

En la Fig. 4 se muestra un esquema del convertidor presentado en [13], definido en este trabajo como Topología C.

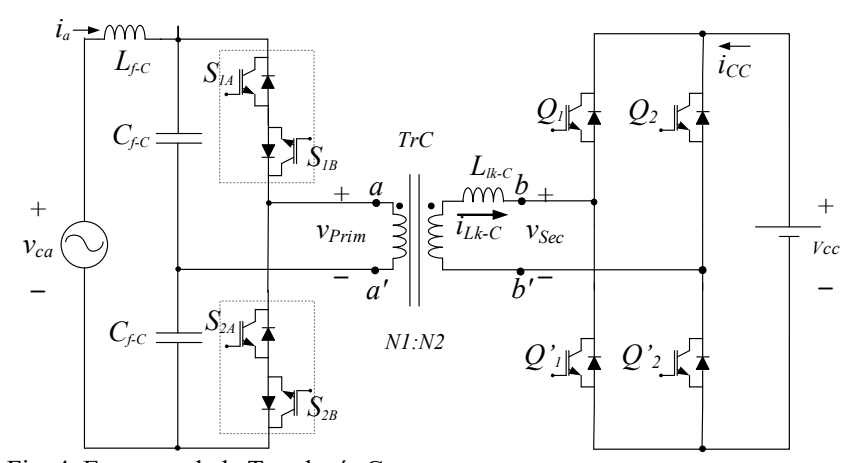

Fig. 4. Esquema de la Topología C.

A diferencia de la topología A, el puente del lado de CA se implementa en una configuración semipuente, en donde una de las piernas está formada por dos llaves bidireccionales en tensión y en corriente, mientras que la otra por un divisor capacitivo. Los puntos medios de ambas piernas están conectados a los bornes de un transformador monofásico de alta frecuencia, $\operatorname{Tr} C$.

El puente del lado de CC se implementa similar a las topologías A y $\mathrm{B}$, descripto anteriormente. La relación de transformación se define como $\mathrm{n}=\mathrm{N} 2 / \mathrm{N} 1, \mathrm{~N} 1$ representa el número de espiras del lado de $\mathrm{CA}$ y $\mathrm{N} 2$ representan el número de espiras del lado de CC.

\section{PRINCIPIO DE FUNCIONAMIENTO}

A continuación se describe el principio de funcionamiento de las topologías indicadas anteriormente, el cual se basa en el mismo principio de los convertidores con puentes duales activos, el cual consiste en controlar el flujo de energía mediante el desfase entre las tensiones a bornes del transformador de alta frecuencia, según se propuso en [14]. En este trabajo el ángulo de desfase se representa con $\delta$.

En las siguientes subsecciones se describe la estrategia de conmutación que debe aplicarse a cada una de las topologías para imponer diferentes niveles de tensiones a bornes de sus respectivos transformadores de alta frecuencia, y luego, se procede a describir la estrategia de modulación propuesta para que las topologías puedan operar con conmutación suave, factor de potencia unitario, con reducida distorsión armónica en la corriente de la red de CA y de manera bidireccional.

\section{A. Estrategia de Conmutación}

Las llaves bidireccionales de cada uno de los puentes de CA se implementan con dos llaves unidireccionales representadas en este trabajo como $S_{x A}$ y su complementaria $S_{x B}$. Mientras que las llaves de cada uno de los puentes de CC se implementan con llaves unidireccionales, representadas en este trabajo como $Q_{X}$, donde $x$ representa el número de la llave semiconductora.

Se establece que las llaves de cada uno de los puentes de CC conmutan de manera complementaria e independientemente de la estrategia de modulación que se utilice.

Con el objetivo de establecer valores de tensión positivos, negativos o nulos, a bornes del transformador de alta frecuencia en función de la polaridad establecida por la regla de los puntos, se establece la secuencia de conmutación que se indica en la Tabla I.

Se debe notar que para el caso de la topología C la tensión en el primario posee una amplitud igual a la mitad de la amplitud de la tensión de red, $v_{\text {Prim }}=v_{c a} / 2$.

\section{B. Estrategia de Modulación}

En esta sección se propone una estrategia de modulación que puede aplicarse a las tres topologías consideradas, la cual consiste en conmutar todas las llaves semiconductoras con un ciclo de trabajo del $50 \%$. En el lado de CC se utiliza una estrategia de modulación por desplazamiento de fase con el fin de modular el ancho de pulso de la tensión impuesta a bornes del transformador en función de la amplitud de la forma de onda de la tensión de red. Por otro lado, las llaves del lado de CA son activadas con un determinado desfase respecto de las 
llaves del lado de CC, el cual es función de la potencia que se desea transferir.

TABLA I

SECUENCIAS DE CONMUTACIÓN

\begin{tabular}{|c|c|c|c|c|c|c|c|c|c|c|c|c|}
\hline \multicolumn{3}{|c|}{$\begin{array}{c}\text { Puente CA } \\
\text { Topología A }\end{array}$} & \multicolumn{3}{|c|}{$\begin{array}{c}\text { Puente CA } \\
\text { Topología B }\end{array}$} & \multicolumn{3}{|c|}{$\begin{array}{l}\text { Puente CA } \\
\text { Topología C }\end{array}$} & \multicolumn{2}{|c|}{ Puente CC } & \multicolumn{2}{|c|}{$\begin{array}{c}\text { Tensiones } \\
\text { del } \\
\text { transformador }\end{array}$} \\
\hline \multicolumn{13}{|c|}{ Si $\boldsymbol{v}_{c a}>\mathbf{0}$} \\
\hline $\begin{array}{l}S_{1 B} \\
S_{4 B}\end{array}$ & $\begin{array}{l}S_{2 \mathrm{~A}}, \\
\mathbf{S}_{3 \mathrm{~A}}\end{array}$ & $\begin{array}{l}\mathbf{S}_{1 \mathrm{~A}}, \\
\mathbf{S}_{2 \mathrm{~B}} \\
\mathbf{S}_{3 \mathrm{~B}} \\
\mathbf{S}_{4 \mathrm{~A}} \\
\end{array}$ & $S_{1 B}$ & $\mathbf{S}_{2 \mathrm{~A}}$ & $\begin{array}{l}S_{1 A} \\
S_{2 B}\end{array}$ & $\mathbf{S}_{1 \mathrm{~A}}$ & $\mathbf{S}_{2 \mathrm{~A}}$ & $\begin{array}{l}S_{1 B} \\
S_{2 B}\end{array}$ & $\mathbf{Q}_{1}$ & $\mathbf{Q}_{2}$ & $v_{\text {Prim }}$ & $v_{S e c}$ \\
\hline 1 & 0 & 1 & 1 & 0 & 1 & 1 & 0 & 1 & 1 & 0 & $+v_{c a}$ & $+V_{c c}$ \\
\hline 0 & 1 & 1 & 0 & 1 & 1 & 0 & 1 & 1 & 0 & 1 & $-v_{c a}$ & $-V_{c c}$ \\
\hline 1 & 0 & 1 & 1 & 0 & 1 & 1 & 0 & 1 & 0 & 0 & $+v_{c a}$ & 0 \\
\hline 0 & 1 & 1 & 0 & 1 & 1 & 0 & 1 & 1 & 1 & 1 & $-v_{c a}$ & 0 \\
\hline \multicolumn{13}{|c|}{ Si $v_{c a}<0$} \\
\hline $\begin{array}{l}\mathbf{S}_{2 \mathrm{~B}}, \\
\mathbf{S}_{3 \mathrm{~B}}\end{array}$ & $\begin{array}{l}\mathbf{S}_{1 \mathrm{~A}}, \\
\mathbf{S}_{4 \mathrm{~A}}\end{array}$ & $\begin{array}{l}\mathbf{S}_{1 \mathrm{~B}} \\
\mathbf{S}_{2 \mathrm{~A}} \\
\mathbf{S}_{3 \mathrm{~A}} \\
\mathbf{S}_{4 \mathrm{~B}}\end{array}$ & $\mathbf{S}_{1 \mathrm{~A}}$ & $\mathbf{S}_{2 B}$ & $\begin{array}{l}\mathbf{S}_{1 \mathrm{~B}} \\
\mathbf{S}_{2 \mathrm{~A}}\end{array}$ & $\mathbf{S}_{1 \mathrm{~B}}$ & $\mathbf{S}_{2 \mathrm{~B}}$ & $\begin{array}{l}\mathbf{S}_{1 \mathrm{~A}} \\
\mathbf{S}_{2 \mathrm{~A}}\end{array}$ & $\mathbf{Q}_{1}$ & $\mathbf{Q}_{2}$ & $v_{\text {Prim }}$ & $v_{S e c}$ \\
\hline 1 & 0 & 1 & 0 & 1 & 1 & 0 & 1 & 1 & 1 & 0 & $+v_{c a}$ & $+V_{c c}$ \\
\hline 0 & 1 & 1 & 1 & 0 & 1 & 1 & 0 & 1 & 0 & 1 & $-v_{c a}$ & $-V_{c c}$ \\
\hline 1 & 0 & 1 & 0 & 1 & 1 & 0 & 1 & 1 & 0 & 0 & $+v_{c a}$ & 0 \\
\hline 0 & 1 & 1 & 1 & 0 & 1 & 1 & 0 & 1 & 1 & 1 & $-v_{c a}$ & 0 \\
\hline
\end{tabular}

En la Fig. 5 se muestra un diagrama en bloques del algoritmo de modulación utilizado. Puede observarse que el cálculo del ancho de pulso $d x$ de la tensión $v_{S e c}$ se realiza a partir de los valores instantáneos de las tensiones $V_{c c}$ y $v_{c a}$. Por otro lado, el ángulo de desfase $\delta$ entre las tensiones $v_{\text {Prim }} \mathrm{y} v_{\text {Sec }}$ se determina a partir de la potencia que se desea transferir. Finalmente, con estos valores se generan las señales PWM de activación de las llaves semiconductoras del convertidor.

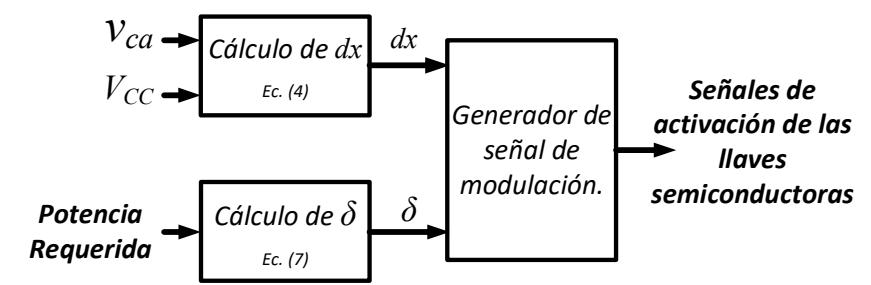

Fig. 5. Diagrama en bloques del algoritmo de modulación implementado.

En la Fig. 6 se muestran conceptualmente las formas de onda de $v_{\text {Prim }}$ y $v_{\text {Sec }}$ para una de las fases, utilizando la estrategia de modulación propuesta.

En la Fig. 7 se muestran las formas de onda de las tensiones y corrientes aplicadas a bornes de $L_{l k} \mathrm{y}$ las áreas $\mathrm{A}$, B y C correspondientes a cada uno de los intervalos resultantes de analizar la caída de tensión aplicada a bornes de $L_{l k}$. El ancho de pulso de la tensión modulada por el puente de $\mathrm{CC}, v_{\mathrm{Sec}}$, está contenida en un medio periodo de la tensión modulada por el puente de CA, $v_{\text {Prim }}$. En este trabajo, a este modo se lo denomina funcionamiento en modo interno.

Con el objetivo de transferir la potencia requerida y de reducir las pérdidas de conmutación [18], las llaves semiconductoras del lado de CA operan con conmutación suave con el principio de ZCS mientras que las llaves del lado de CC operan con conmutación suave con el principio de ZVS.

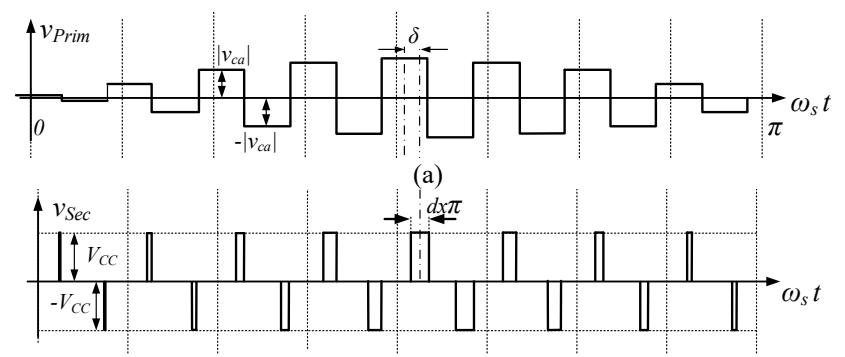

(b)

Fig. 6. Formas de ondas de las tensiones impuestas a bornes del transformador de alta frecuencia, utilizando la estrategia de modulación propuesta durante medio ciclo de la tensión de red. (a) Tensión del lado de CA, vrim.(b) Tensión del lado de CC, $v_{\text {Sec }}$.

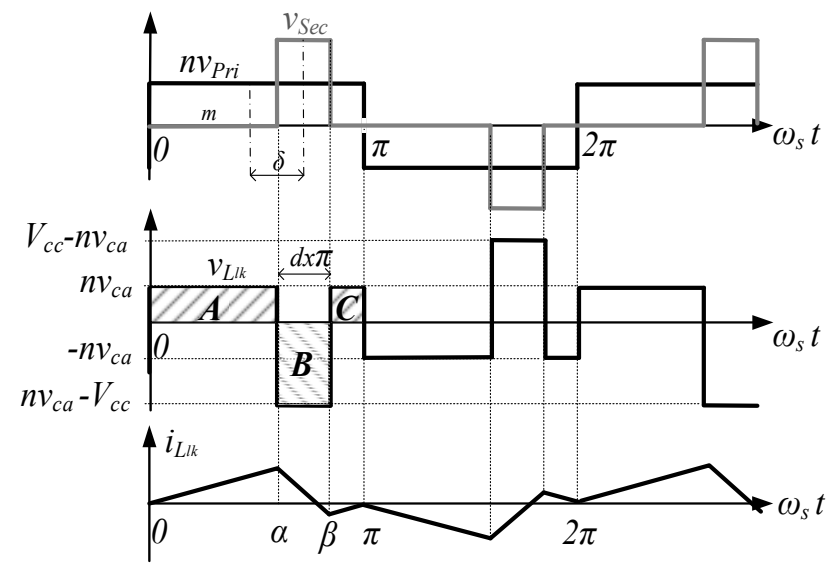

Fig. 7. Modulación para reducir pérdidas por conmutación. (ZCS) (ZVS).

Para ello, se establece que la corriente inicial de la inductancia de dispersión equivalente del transformador sea nula y que en régimen permanente su caída de tensión sea nula, como se muestra en la Fig. 7, cumpliéndose la siguiente condición.

$$
\int_{0}^{\pi} v_{L k} d \omega_{s} t=\sum \text { Areas }=A+B+C=0
$$

donde $V_{L k}$ es la tensión aplicada a bornes de $L_{l k}, \omega_{s}=2 \pi f_{s}$ y $f_{s}$ es la frecuencia de conmutación.

A partir de la Fig. 7 pueden plantearse las ecuaciones correspondientes a las áreas A, B y C como:

$$
\begin{aligned}
& A=n\left|\nu_{c a}\right|\left(\frac{\pi}{2}+\delta-d x \frac{\pi}{2}\right) \\
& B=\left(n\left|v_{c a}\right|-V_{c c}\right) d x \pi \\
& C=n\left|v_{c a}\right|\left(\frac{\pi}{2}-\delta-d x \frac{\pi}{2}\right)
\end{aligned}
$$

Reemplazando los valores de A, B y C indicados en (7) en (6) y resolviendo, puede deducirse la expresión del ciclo de trabajo $d x$, de la tensión del lado de CC, como 


$$
d x=\frac{n\left|v_{c a}\right|}{V_{c c}}
$$

Para la topología A se considera el valor pico de $v_{c a}=\hat{V}_{c a}$ entonces el valor máximo de $d x_{\max }=n V_{c a} \mathrm{I} / / V_{c c}$. En el caso de la topología $B$ se reemplaza el valor de $n$ por $n_{b}$, siendo $\mathrm{n}_{\mathrm{b}}=2 \mathrm{n}$. Por último, en el caso de la topología $\mathrm{C}$ se debe tener en cuenta que $v_{c a}=V_{c a} / 2$.

A su vez $d x_{\max }$ limita el ángulo de desfase a un valor máximo igual a

$$
\delta_{\max }=\frac{\pi}{2}\left(1-d x_{\max }\right),
$$

donde $\delta_{\max }$ es el máximo valor que puede tomar $\delta$ en función de $d x_{\max }$ para operar en modo interno.

Con esto queda establecido que para operar cada una de las topologías estudiadas en este trabajo en modo interno, debe determinarse un valor máximo de $d x$ el cual establece el valor de desfase $\delta$ máximo que puede aplicarse. Esta condición establece que existe una potencia máxima que puede transferirse con cada una de las topologías estudiadas como será analizado posteriormente en este trabajo.

\section{Determinación de la Potencia Media de Cada Topología}

Utilizando la estrategia de modulación indicada anteriormente, puede calcularse la potencia media $\left(P_{m e d}\right)$ transferida por cada topología con la siguiente expresión

$$
P(x)_{\text {med }}=\frac{1}{\pi} \int_{0}^{\pi}\left(\frac{1}{\pi} \int_{0}^{\pi} v_{S e c} i_{L k} d \omega_{s} t\right) d \omega_{r} t
$$

donde x corresponde a la topología, $I_{L k}$ la corriente en $L_{l k}$.

Resolviendo la $P(x)_{\text {med }}$ para cada una de las topologías presentadas se pueden obtener las siguientes expresiones:

$$
\begin{gathered}
P(A)_{\text {med }}=\frac{n^{2} V_{c a}{ }^{2}|\delta|}{4 \pi f_{s} L_{l k-A}} \\
P(B)_{\text {med }}=\frac{n^{2} V_{c a}{ }^{2}|\delta|}{\pi f_{s} L_{l k-B}} \\
P(C)_{\text {med }}=\frac{n^{2} V_{c a}{ }^{2}|\delta|}{16 \pi f_{s} L_{l k-C}}
\end{gathered}
$$

En la Fig. 8 se muestra la variación de las potencias expresadas en (7) en función del ángulo de desfase $\delta$, para un intervalo entre $+\pi / 4[\mathrm{rad}]$ y $-\pi / 4$ [rad]. Puede observarse que para valores positivos de $\delta$ la potencia es positiva, transfiriéndose potencia desde la red de CA hacia la red de $\mathrm{CC}$, y para valores negativos de $\delta$ la potencia es negativa, transfiriéndose potencia desde la red de CC hacia la red de CA.

Para obtener una $P_{\text {med }}=5 \mathrm{~kW}$ con un desfase $\delta=\pi / 6$ [rad] se calcularon los valores de $L_{l k}$, utilizando las ecuaciones (7), dando como resultado $L_{l k-A}=10 \mu \mathrm{H}$ para la Topología A, $L_{l k-B}=40.3 \mu \mathrm{H}$ para la Topología B y $L_{l k-C}=2.52 \mu \mathrm{H}$ para la Topología C. Se utilizó como criterio que las tres topologías transfieran la misma potencia máxima y de esta manera establecer los valores de inductancia de dispersión equivalente necesarios para cada topología.

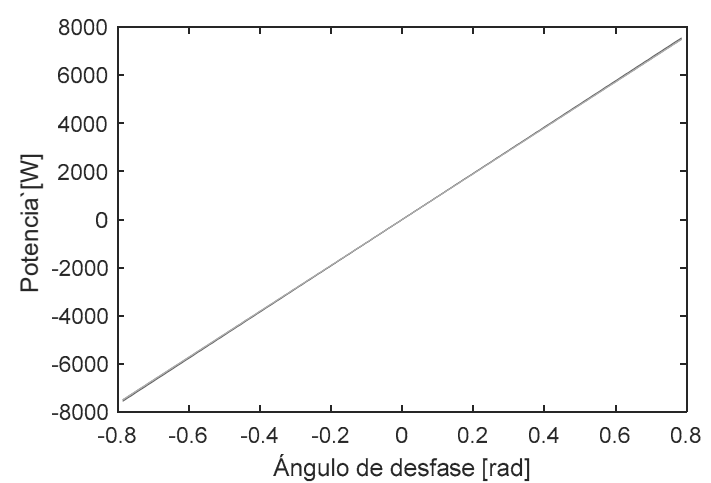

Fig. 8. Potencia transferida por cada convertidor en función del ángulo de desfase $\delta$.

\section{Diseño del Filtro $L C$}

A continuación, se presentan las expresiones para determinar los valores del inductor $L_{f}$ y el capacitor $C_{f}$ que componen al filtro LC del lado de CA.

La expresión para determinar el ripple de tensión del capacitor puede escribirse como:

$$
\Delta v_{c}=\frac{\Delta Q_{c}}{C_{f}}
$$

donde $\Delta Q_{c}$ es la carga recibida por el capacitor y $C_{f}$ es el valor de su capacidad.

La corriente a través del capacitor durante cada medio periodo de conmutación es la diferencia entre la corriente del transformador, $i_{L k}$, y la corriente de fase filtrada, $i_{c a}$. De esta manera, la expresión para determinar la ondulación de tensión para cada una de las topologías puede escribirse como:

$$
\begin{aligned}
\Delta v_{A \text { (Topologia }-A)} & =\frac{1}{C_{f-A}} \int_{0}^{\frac{T_{s}}{2}}\left(n i_{L k-A}-i_{c a}\right) d t \\
& =\frac{n^{3} V_{c a}{ }^{2} \pi^{2}\left|\sin \left(\omega_{r} t\right)\right|^{2}\left(V_{C C}-n V_{c a}\left|\sin \left(\omega_{r} t\right)\right|\right)}{16 \pi^{2} f_{s}^{2} C_{f-A} L_{f-A} V_{C C}{ }^{2}} \\
\Delta v_{B(\text { Topologia }-B)} & =\frac{1}{C_{f-B}} \int_{0}^{\frac{T_{s}}{2}}\left(n i_{L k-B}-i_{c a}\right) d t \\
& =\frac{n^{3} V_{c a}{ }^{2} \pi^{2}\left|\sin \left(\omega_{r} t\right)\right|^{2}\left(V_{C C}-2 n V_{c a}\left|\sin \left(\omega_{r} t\right)\right|\right)}{4 \pi^{2} f_{s}{ }^{2} C_{f-B} L_{f-B} V_{C C}{ }^{2}} \\
\Delta v_{C \text { (Topologia }-C)} & =\frac{1}{C_{f-C}} \int_{0}^{\frac{T_{s}}{2}}\left(n i_{L k-C}-i_{c a}\right) d t \\
& =\frac{n^{3} V_{c a}{ }^{2} \pi^{2}\left|\sin \left(\omega_{r} t\right)\right|^{2}\left(2 V_{C C}-n V_{c a}\left|\sin \left(\omega_{r} t\right)\right|\right)}{128 \pi^{2} f_{s}{ }^{2} C_{f-C} L_{f-C} V_{C C}{ }^{2}}
\end{aligned}
$$

donde $\Delta t$ es el desplazamiento temporal, el cual es función del ángulo de desfase $\delta, T_{s}$ es el periodo de conmutación, $n$ es la 
relación de conversión del transformador, $V_{c a}$ es el valor pico de la tensión de la red de CA, $\omega_{r}=2 \pi f_{r}$, y $L_{x}$ es la inductancia de dispersión del transformador.

De las ecuaciones anteriores, el valor de $C_{f}$ para cada topología puede determinarse en función del valor de la ondulación de tensión deseada. Considerando que el mayor valor ocurre cuando $\left|\sin \left(\omega_{r} t\right)\right|=1$ y estableciendo un valor máximo de ondulación de $30 \mathrm{~V}$, evaluando las ecuaciones (9), (10) y (11) se obtienen los siguientes valores:

$C_{f-A}=7.7 \mu \mathrm{F}, C_{f-B}=5 \mu \mathrm{F}$ y $C_{f-C}=9 \mu \mathrm{F}$.

El filtro LC es diseñado para eliminar las componentes de alta frecuencia en las corrientes inyectadas a la red. El valor de $C_{f}$ fue definido en función de la ondulación de tensión deseada. Asumiendo una frecuencia de corte $f_{c}=2 \mathrm{kHz}$, el valor de $L_{f}$ para cada topología está determinado por,

$$
\begin{aligned}
& L_{f-A}=\frac{1}{\left(2 \pi f_{c}\right)^{2} C_{f-A}}=822 \mu \mathrm{H}, \\
& L_{f-B}=\frac{1}{\left(2 \pi f_{c}\right)^{2} C_{f-B}}=1.3 \mathrm{mH}, \\
& L_{f-C}=\frac{1}{\left(2 \pi f_{c}\right)^{2} C_{f-C}}=703 \mu \mathrm{H} .
\end{aligned}
$$

\section{RESULTADOS DE SIMULACIÓN Y COMPARACIÓN DE LAS TOPOLOGÍAS}

Con los objetivos de comparar y validar el funcionamiento de las topologías, en esta sección se presentan resultados de simulación utilizando transformadores de alta frecuencia y llaves semiconductoras ideales, considerando los parámetros indicados en la TABLA II. Los resultados de simulación fueron obtenidos mediante la implementación de los SST en el software PSIM. Los módulos de control se programaron en lenguaje $\mathrm{C}$.

TABLA II

PARÁMETROS DE SIMULACIÓN

\begin{tabular}{l|l|l|l|l|l}
\multicolumn{6}{c}{ PARÁMETROS DE SIMULACIÓN } \\
\hline \hline$V_{C C}$ & $V_{c a}$ & $f_{r}$ & $f_{s}$ & $n$ & $P_{o}$ \\
\hline $600 \mathrm{~V}$ & $311 \mathrm{~V}$ & $50 \mathrm{~Hz}$ & $20 \mathrm{kHz}$ & 0.5 & $7.5 \mathrm{~kW}$ \\
\hline \hline
\end{tabular}

Para cada una de las topologías consideradas se obtuvieron valores de $d x_{\max }$ y $\delta_{\max }$ mostrados en TABLA III correspondientes al límite para el funcionamiento en modo interno. Los resultados de simulación que se presentan a continuación fueron realizados para transferir una potencia media aproximada de $5 \mathrm{~kW}$ y $-5 \mathrm{~kW}$, con un desfase de $\delta=\pi / 6$ [rad] y $\delta=-\pi / 6$ [rad], respectivamente, con lo que se demuestra la bidireccionalidad de los convertidores evaluados en el presente trabajo.

\begin{tabular}{|c|c|c|c|}
\hline & Topologia A & Topologia B & Topologia C \\
\hline$d x_{\max }$ & 0.2591 & 0.5183 & 0.1296 \\
\hline$\delta_{\max }$ & $\pm 1.16[\mathrm{RAD}]\left(66.6^{\circ}\right)$ & $\pm 0.75[\mathrm{RAD}]\left(43.3^{\circ}\right)$ & $\pm 1.36[\mathrm{RAD}]\left(78.3^{\circ}\right)$ \\
\hline
\end{tabular}

TABLA III

LÍMITES DE FUNCIONAMIENTO EN MODO INTERNO
E. Resultados de Simulaciones Correspondientes a las Topologías $A$, $B$ y $C$

A continuación, se muestran los resultados obtenidos para cada una de las Topologías, en los cuales pueden observarse las formas de onda de las tensiones $\mathrm{n} v_{\text {Prim }} \mathrm{y} v_{\text {Sec }}$, la corriente $I_{l k}$ y las tensiones y corrientes de las llaves semiconductoras del lado de CA (V_llaveCA, I_llave_CA) y del lado de CC (V_llaveCC, I_llave_CC). Las Fig. 9, 10 y 11 corresponden a las simulaciones de las Topologías A, B y $\mathrm{C}$ respectivamente.
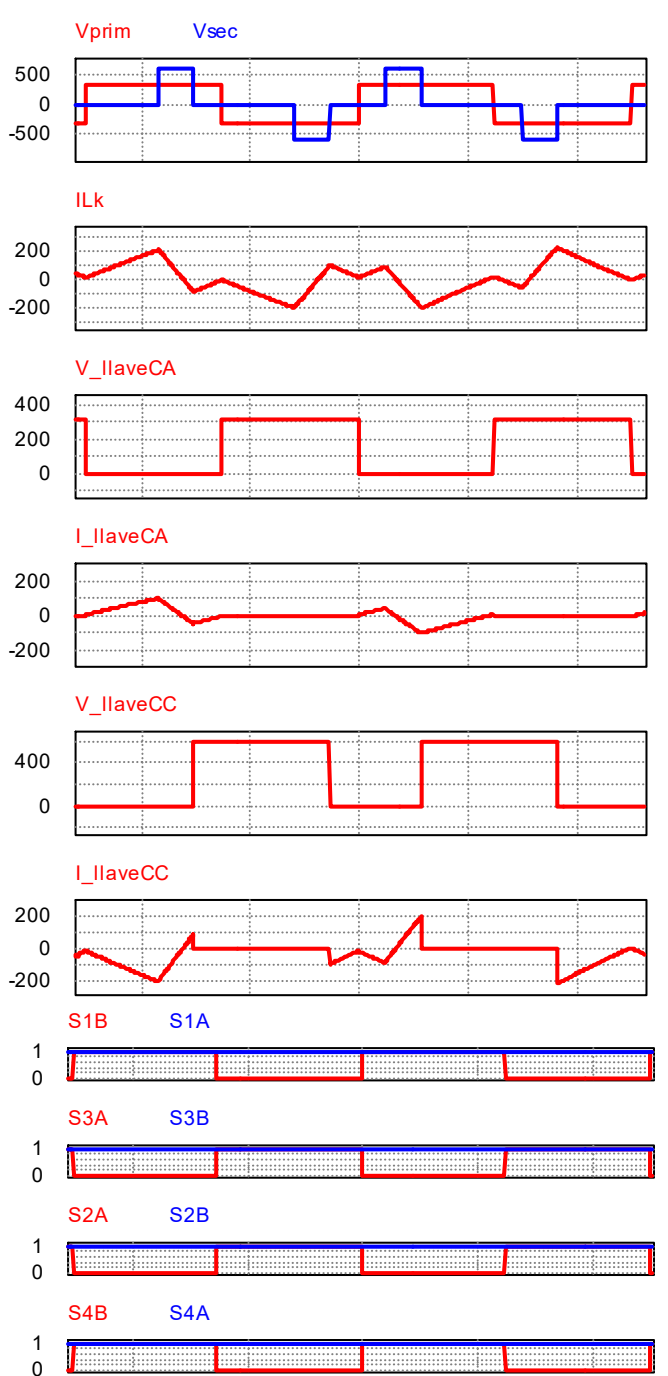

1

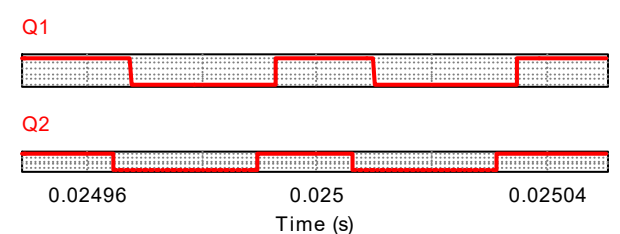

Fig. 9. Resultados de simulación de corrientes y tensiones en las llaves (Topología A). Referencias de potencia: $\mathrm{P}(0 \mathrm{~s})=5 \mathrm{~kW}, \mathrm{P}(25 \mathrm{~ms})=-5 \mathrm{~kW}$.

En estas figuras puede observarse que hasta los $25 \mathrm{~ms}$ se transfieren $5 \mathrm{~kW}$ desde la red de CA hacia la red de CC, con un desfase $\delta=\pi / 6$ [rad] entre $v_{\text {Prim }}$ y $v_{\text {Sec }}$. Luego de los $25 \mathrm{~ms}$ la referencia de potencia cambia a $-5 \mathrm{~kW}$, y la potencia es 
transferida desde la red de $\mathrm{CC}$ hacia la red de $\mathrm{CA}$, con un desfase $\delta=-\pi / 6[\mathrm{rad}]$ entre $v_{\text {Prim }} \mathrm{y} v_{\text {Sec }}$.

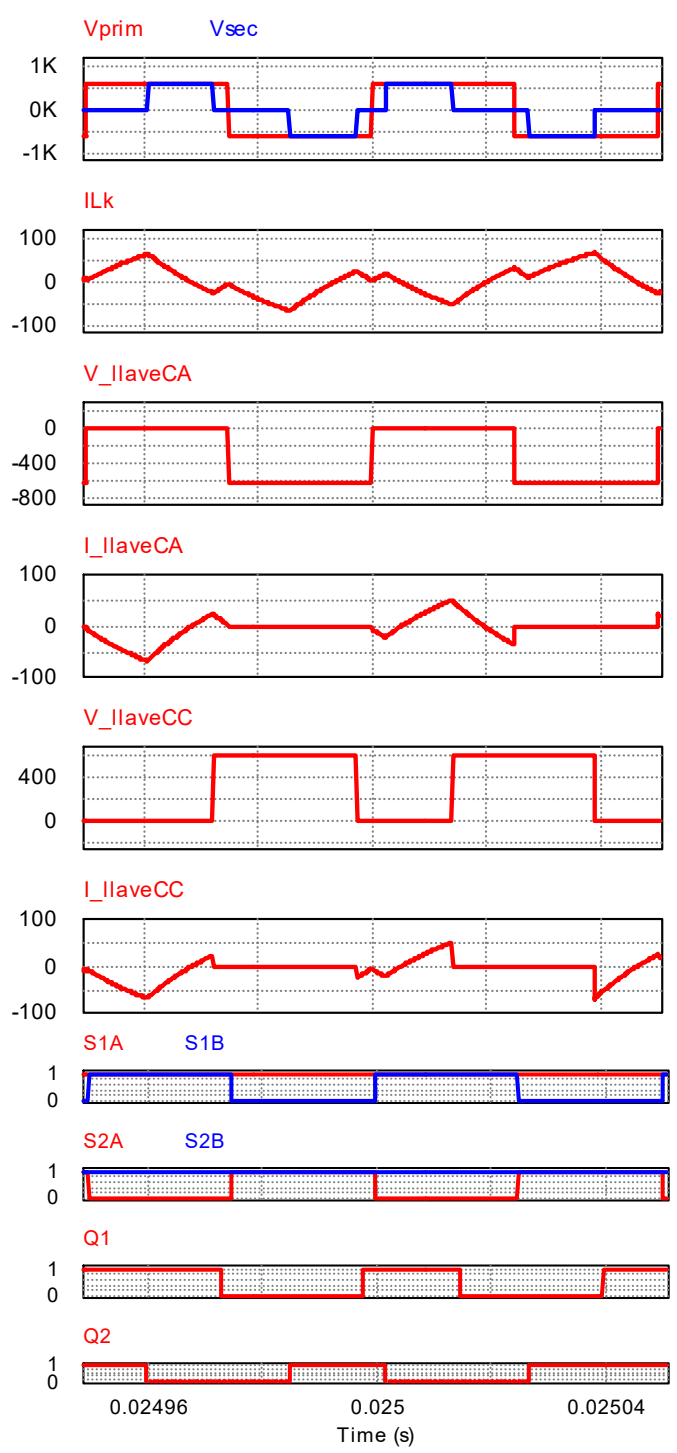

Fig. 10. Resultados de simulación de corrientes y tensiones en las llaves (Topología B). Referencias de potencia: $\mathrm{P}(0 \mathrm{~s})=5 \mathrm{~kW}, \mathrm{P}(25 \mathrm{~ms})=-5 \mathrm{~kW}$.

Por otro lado, puede observarse que las llaves bidireccionales de los puentes del lado de CA $\left(S_{j A}, S_{j B}\right.$, con $j=1,2,3,4)$ se activan y desactivan en los ángulos 0 [rad] y $\pi$ [rad], respectivamente, cuando la corriente del inductor se anula, demostrándose que estas llaves operan con ZCS. Además, debido a que las llaves semiconductoras del puente del lado de CC $\left(Q_{j}, Q_{j}^{\prime}\right)$ conmutan en los ángulos $\alpha$ y $\beta$, y durante estos instantes la corriente circula a través de sus respectivos diodos en antiparalelo, la conmutación se realiza con ZVS.

Los valores picos de las corrientes en las llaves del puente de CA presentan valores próximos a 100A para la Topología A, 60A para la Topología B y 200A para la Topología C. Por otro lado, las llaves semiconductoras del puente de CC presentan valores de corriente pico próximos a $200 \mathrm{~A}$ para la Topología A, 60A para la Topología B y 500A para la
Topología C. Con estos resultados se puede mencionar que las llaves que deben soportar mayor corriente son las de la topología $\mathrm{C}$ y las que deben soportar menor corriente son las de la topología B.

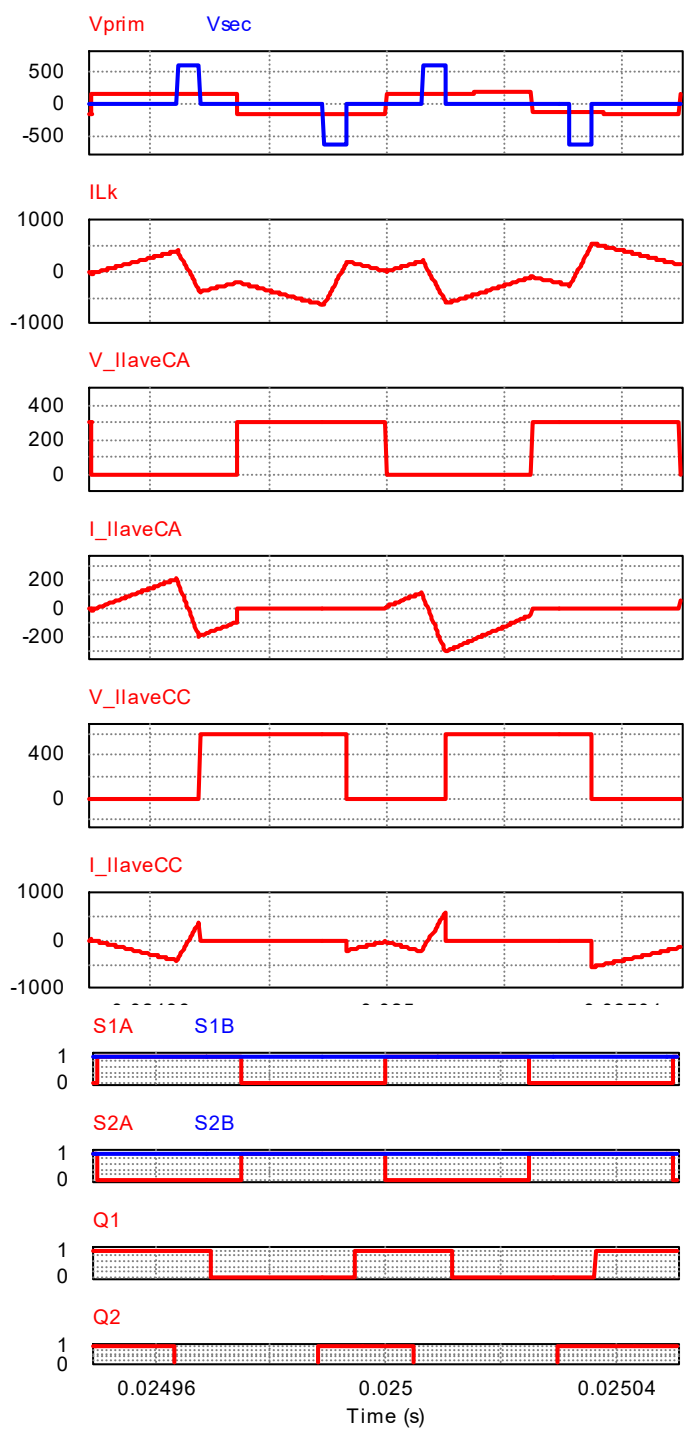

Fig. 11. Resultados de simulación de corrientes y tensiones en las llaves (Topología C). Referencias de potencia: $\mathrm{P}(0 \mathrm{~s})=5 \mathrm{~kW}, \mathrm{P}(25 \mathrm{~ms})=-5 \mathrm{~kW}$.

Las tensiones en las llaves del puente de CA presentan valores máximos de $311 \mathrm{~V}, 622 \mathrm{~V}$ y $311 \mathrm{~V}$ correspondientes a las Topologías A, B y C, siendo las llaves de la topología B las que deben soportar mayores tensiones. Las tensiones en las llaves del puente de CC presentan valores máximos de $600 \mathrm{~V}$ en las tres Topologías.

La Fig. 12 presenta las formas de onda, en ocho períodos de frecuencia de red, de la tensión de línea $\left(v_{c a}\right)$ y las corrientes de linea $\left(i_{c a}\right)$ del lado del puente de CA para cada una de las topologías presentadas. Puede observarse que desde el tiempo inicial $\mathrm{t}=0 \mathrm{~s}$ hasta los $40 \mathrm{~ms}$ la referencia de potencia es de $5 \mathrm{~kW}$, luego desde los $40 \mathrm{~ms}$ hasta los $80 \mathrm{~ms}$ la referencia de potencia es de $2.5 \mathrm{~kW}$, en ambos casos el sentido de transferencia de potencia es desde la red de CA hacia la red de 
CC. A los $80 \mathrm{~ms}$ se realiza un cambio en la referencia de potencia, desde $2.5 \mathrm{~kW}$ a $-2.5 \mathrm{~kW}$, con lo cual el sentido de transferencia de potencia pasa a ser desde la red de CC hacia la red de CA. Desde los $80 \mathrm{~ms}$ hasta los $120 \mathrm{~ms}$ la referencia de potencia es de $-2.5 \mathrm{~kW}$, luego desde los $120 \mathrm{~ms}$ hasta los $160 \mathrm{~ms}$ la referencia de potencia es de $-5 \mathrm{~kW}$. Con estos resultados se muestra el comportamiento de los convertidores ante cambios de referencia de potencia y la bidireccionalidad de los mismos.

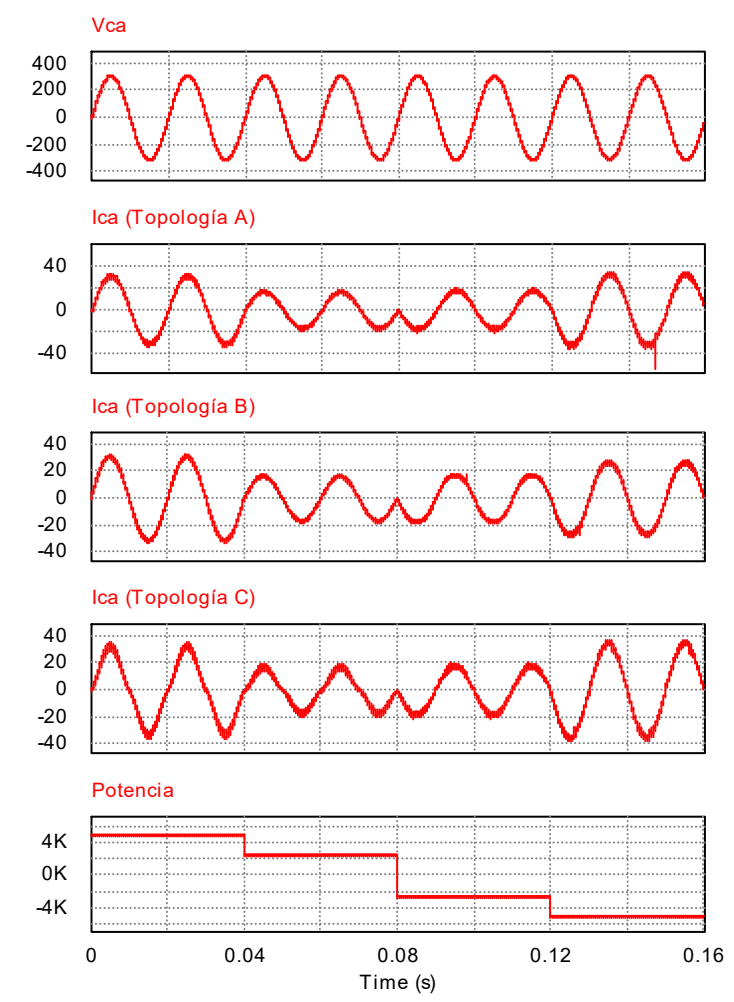

Fig. 12. Resultados de simulación de la tensión de línea (Vca) y la corriente de línea (Ica) para las tres topologías. Referencias de potencia: $\mathrm{P}(0 \mathrm{~s})=5 \mathrm{~kW}$, $\mathrm{P}(40 \mathrm{~ms})=2.5 \mathrm{~kW}, \mathrm{P}(80 \mathrm{~ms})=-2.5 \mathrm{~kW}, \mathrm{P}(120 \mathrm{~ms})=-5 \mathrm{~kW}$.

\section{F. Comparación de los Resultados de las Topologías}

A fin de comparar el desempeño de cada topología se evaluó el factor de potencia $(F P)$, la distorsión armónica total en las corrientes de CA (THDi) y el factor de utilización $(F U)$, de las llaves semiconductoras para el rango de desfase $\delta= \pm 0.75[\mathrm{rad}]\left(43.3^{\circ}\right)$.

El THDi de las corrientes de fase se calculó utilizando la siguiente expresión [21].

$$
T H D_{i}=\frac{\sqrt{I_{r m s}{ }^{2}-I_{1}^{2}}}{I_{1}}
$$

donde $I_{r m s}$ es el valor eficaz de la corriente de fase y $I_{l}$ es el valor rms de la componente fundamental de la corriente.

En la Fig. 13 se muestra la variación del THDi en función de $\delta$ para cada una de las topologías. Se observa que solo para valores de $|\delta|>10^{\circ}$ presentan valores por debajo al $5 \%$. Estos valores son aceptables, teniendo en cuenta los valores máximos de distorsión que establece la norma IEEE-1547.
Para valores de $|\delta|<10^{\circ}$, con la topología A se alcanzan los valores máximos de THDi y con la topología $\mathrm{C}$ se alcanzan los valores mínimos.

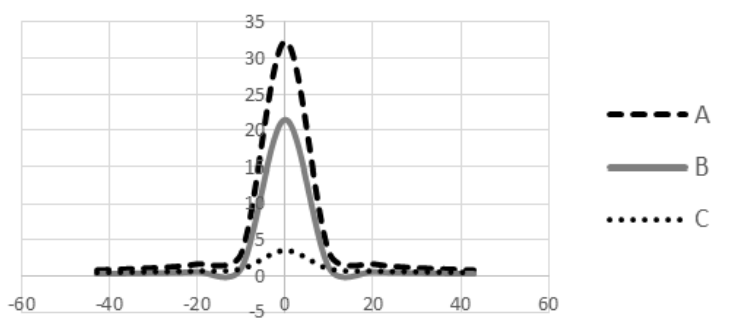

Fig. 13. Variación de $T H D i$ en función de $\delta$ para cada una de las topologías.

El FP se calculó usando la siguiente expresión [22]:

$$
F P=\frac{P_{C A}}{S_{C A}}=\frac{\cos \theta}{\sqrt{1+T H D_{i}^{2}}}
$$

donde $P_{C A}$ es la potencia activa y $S_{C A}$ es la potencia aparente que el convertidor transfiere hacia la red de CA.

En la Fig. 14 se muestra la variación del $F P$ en función de $\delta$. Se puede observar que las topologías A y B presentan valores muy similares, cercanos a 0.99 para $|\delta|>10^{\circ}$ y para valores de $|\delta|<10^{\circ}$ se obtuvieron valores de $F P$ por encima de 0.94 y 0.97 respectivamente. La topología $C$ es la que presenta mayor variación, obteniéndose $F P$ por encima de 0.92 para $|\delta|>20^{\circ}$ y decrecientes hasta alcanzar el mínimo de 0.11 para $|\delta|<20^{\circ}$.

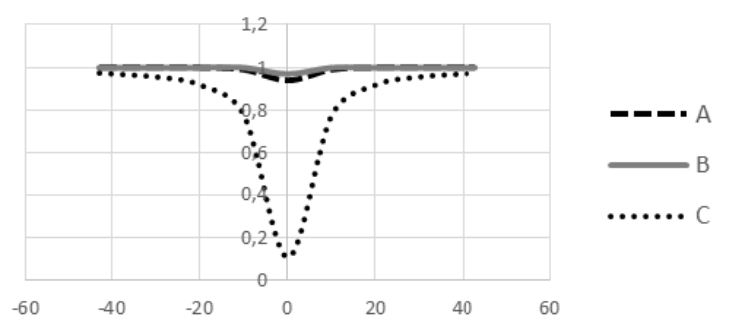

Fig. 14. Variación del $F P$ en función de $\delta$ para cada topología.

Como puede observarse en los resultados obtenidos, para transferir una misma potencia, la topología B utiliza un mayor valor de inductancia de dispersión, con lo cual los valores de corriente son menores en comparación a las corrientes que deben manejar las otras topologías, presentando en consecuencia menores valores picos de corriente, menor distorsión armónica y mayor factor de potencia.

Para evaluar el $F U$ de los interruptores del convertidor para cada topología se utilizó la siguiente expresión [22],

$$
F U=\frac{P_{\text {med }}}{S}
$$

Siendo $S$ el factor de estrés de cada llave semiconductora. $\mathrm{Si}$ el puente del lado de CA o de CC contiene $\mathrm{k}$ llaves entonces el factor de estrés en cada puente queda definido como:

$$
S(\text { puente })=\sum_{x=1}^{k} I_{r m s}-\mathrm{S}_{(\mathrm{x})} \mathrm{V}_{\text {pico }-} \mathrm{S}_{(\mathrm{x})}
$$


donde $I_{r m s} \quad S_{(x)}$ es la corriente rms que maneja la llave (x) en un período de red, $V_{\text {pico_ }} s_{(x)}$ es la tensión pico que maneja la misma llave en un período de red y $(\mathrm{x})$ corresponde al número de llave pertenecientes al puente en el lado de CA o en el lado de CC.

En la Fig. 15 se muestran los resultados de evaluar el $F U$ de las llaves bidireccionales y el $F U$ de las llaves unidireccionales con diodos en antiparalelo de cada una de las topologías, todos en función del desfase $\delta$. Se observa que los valores de $F U$ más elevados se muestran en las llaves bidireccionales de la topología $\mathrm{A}$, y los más reducidos en las llaves unidireccionales de la topología $\mathrm{C}$.

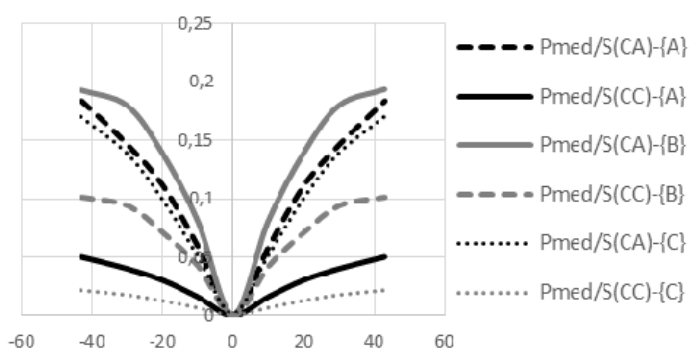

Fig. 15. Variación del $F U$ en función de $\delta$ para las llaves del lado de CC y CA de cada topología.

En la TABLA IV se muestra un resumen de las características de cada convertidor.

TABLA IV

RESUMEN DE CADA CONVERTIDOR

\begin{tabular}{cccc}
\hline \hline Característica & Topología $\boldsymbol{A}$ & Topología $\boldsymbol{B}$ & Topología $\boldsymbol{C}$ \\
\hline$N^{o}$ llaves bidireccionales & 4 & 2 & 2 \\
$N^{o}$ llaves unidireccionales & 4 & 4 & 4 \\
$F U_{\operatorname{máx}}$ & 0.2679 & 0.2566 & 0.0873 \\
$F P_{\operatorname{máx}}$ (con filtro) & 0.9993 & 0.993 & 0.977 \\
$T H D_{i, \min \text { (con filtro) }}$ & $0.83 \%$ & $0.30 \%$ & $0.52 \%$ \\
\hline \hline
\end{tabular}

\section{CONCLUSiones}

Se analizaron y compararon tres topologías de SST monofásicos, CC-CA, bidireccionales, aislados, elevadores y reductores de tensión para aplicaciones en microrredes eléctricas.

Los convertidores estudiados presentan componentes armónicas de alta frecuencia, por lo cual se necesita de un filtro para atenuarlas y reducir el valor de THDi de las corrientes.

Se propuso una estrategia de modulación, aplicable a las tres topologías estudiadas, que permite reducir los requerimientos del filtro y que además permite controlar la transferencia de potencia con bajas pérdidas de conmutación, debido a que las llaves semiconductoras operan con conmutación suave en todo su rango de funcionamiento.

Se realizaron simulaciones numéricas para validar el desempeño de cada una de las topologías analizadas, las cuales fueron diseñadas para tener la capacidad de transferir la misma potencia.

Los resultados obtenidos, muestran un valor de THDi inferior al 5\% para las tres topologías. Sin embargo, la topología B es la que presenta el menor THDi de todas.
Los valores máximos del $F P$ alcanzados fueron de 0.99 para las topologías A y B, y 0.97 para la topología C.

Con respecto al $F U$ de las llaves semiconductoras del puente de CA alcanzan un valor máximo próximo a 0.267 en la topología A, 0.256 en la topología B y 0.087 en la topología C. En el caso del puente de CC las llaves alcanzan un valor máximo de $F U$ próximo a 0.069 para la topología A, 0.132 para la topología B y 0.022 para la topología C.

En relación a las corrientes pico a través de las llaves de los puentes de CA y de CC, la topología $\mathrm{B}$ presenta los valores más reducidos seguidos por las Topologías A y C.

Las tensiones máximas soportadas por las llaves en el puente de CA presentan los valores más elevados en la topología B, duplicando a los valores de las Topologías A y C. Las tensiones soportadas en las llaves del puente de CC son iguales en las tres Topologías.

De acuerdo a los resultados obtenidos, la topología B resulta ser la opción que brinda mejor calidad de energía al momento de intercambiar potencia entre la microrred de CC y la red de CA convencional, ya que con la misma se obtienen menores valores de THDi, mayores valores de FP, y por otro lado los valores picos de corriente que deben manejar las llaves semiconductoras son menores.

Este trabajo se ha limitado a evaluar cuantitativamente la calidad de energía y el estrés de las llaves semiconductoras de los convertidores presentados. Como trabajos futuros se pretende analizar el rendimiento, la capacidad de compensación de potencia reactiva y obtener resultados experimentales con cada una de estas topologías.

\section{AGRADECIMIENTOS}

Este trabajo fue apoyado por las siguientes agencias argentinas: Secretaría de Ciencia y Técnica de la Universidad Nacional de Río Cuarto (SeCyT, UNRC), Consejo Nacional de Investigaciones Científicas y Técnicas (CONICET), Agencia Nacional de Promoción Científica y Tecnológica (FONCyT) a través del proyecto PICT 1663/2016 y el Ministerio de Ciencia y Tecnología de la Provincia de Córdoba a través del proyecto FONTEC 2018 "I + D de transformador de estado sólido para aplicaciones rurales".

\section{REFERENCIAS}

[1] W. W. Weaver, R. D. Robinett Iii, G. G. Parker, and D. G. Wilson, "Energy storage requirements of dc microgrids with high penetration renewables under droop control," International Journal of Electrical Power \& Energy Systems, vol. 68, pp. 203-209, 2015.

[2] A. Khaligh and Z. Li, "Battery, Ultracapacitor, Fuel Cell, and Hybrid Energy Storage Systems for Electric, Hybrid Electric, Fuel Cell, and Plug-In Hybrid Electric Vehicles: State of the Art," IEEE Transactions on Vehicular Technology, vol. 59, pp. 2806-2814, 2010.

[3] M. Elsied, A. Oukaour, H. Gualous, and R. Hassan, "Energy management and optimization in microgrid system based on a green energy," Energy, vol. 84, pp. 139-151, 2015.

[4] J.M. Carrasco, L.G. Franquelo, J.T. Bialasiewicz, E. Galvan, R.C.P. Guisado, Ma.A.M Prats, J.I. Leon, N. Moreno-Alfonso. "PowerElectronic Systems for the Grid Integration of Renewable Energy Sources: A Survey". IEEE Trans. on Ind. Electron.; vol. 53, no. 4, pp. $1002-1016,2006$.

[5] F. Blaabjerg, C. Zhe, S. B. Kjaer. "Power electronics as efficient interface in dispersed power generation systems." IEEE Trans. on Power Electronics, vol. 19, no.5, pp. 1184-1194, 2006.

[6] S. Xu, A. Q. Huang, and R. Burgos, "Review of Solid-State Transformer Technologies and Their Application in Power Distribution 
Systems", IEEE Journal of Emerging and Selected Topics in Power Electronics, vol. 1, pp. 186-198, 2013.

[7] M. Liserre, G. Buticchi, M. Andresen, G. De Carne, L. F. Costa, Z. X Zou. "The Smart Transformer: Impact on the Electric Grid and Technology Challenges." IEEE Industrial Electronics Magazine 10(2): 46-58. 2016

[8] J. W. Kolar, G. I. Ortiz. "Solid State Transformer Concepts in Traction and Smart Grid Applications". Proceedings of the 28th Applied Power Electronics Conference and Exposition (APEC 2013), Long Beach, California, USA, March 17-21, 2013.

[9] Z. X. Zou, G. Buticchi, and M. Liserre, "Control and communication in the Smart Transformer-fed grid," 21st International Conference on Emerging Technologies and Factory Automation (ETFA), 2016, pp. 19.

[10] N. D. Weise, G. Castelino, K. Basu and N. Mohan. "A Single-Stage Dual-Active-Bridge-Based Soft Switched AC-DC Converter with Open-Loop Power Factor Correction and Other Advanced Features." IEEE Trans. on Power Electronics. vol. 29, no. 8, pp. 4007-4016, 2014.

[11] R. N. Beres, X. Wang, M. Liserre, F. Blaabjerg, C. L. Baket. "A Review of Passive Power Filters for Three-Phase Grid-Connected Voltage-Source Converters." IEEE Journal of Emerging and Selected Topics in Power Electronics 4(1): 54-69. 2016.

[12] G. Castelino, K. Basu, N. Weise and N. Mohan. "A bi-directional, isolated, single-stage, DAB-based AC-DC converter with open-loop power factor correction and other advanced features". IEEE International Conference on Industrial Technology (ICIT), 2012.

[13] F. Jauch, and J. Biela. "Combined Phase Shift and Frequency Modulation of a Dual Active Bridge AC-DC Converter with PFC." Power Electronics, IEEE Transactions on PP(99): 1-1. 2016.

[14] W. A. A. De Doncker, D. M. Divan, M. H. Kheraluwala. "A threephase soft-switched high-power-density DC/DC converter for highpower applications". IEEE Transactions on Industry Applications, vol. 27, no. 1, January/February 1991

[15] A. K. Jain, and R. Ayyanar, "Pwm Control of Dual Active Bridge: Comprehensive Analysis and Experimental Verification", IEEE Transactions on Power Electronics, 26, 1215-27, 2011.

[16] F. Krismer, and J. W. Kolar. "Closed Form Solution for Minimum Conduction Loss Modulation of Dab Converters." Power Electronics, IEEE Transactions on 27, no. 1, 174-188. 2012.

[17] K. Vangen, T. Melaa, and A. K. Adnanes. "Soft-Switched HighFrequency, High Power Dc/Ac Converter with Igbt." In Power Electronics Specialists Conference, 1992. PESC '92 Record., 23rd Annual IEEE, 26-33 vol.1, 1992.

[18] G. G. Oggier, G. O. Garcia, and A. R. Oliva, "Switching Control Strategy to Minimize Dual Active Bridge Converter Losses," IEEE Transactions on Power Electronics, vol. 24, pp. 1826-1838, 2009.

[19] T. Haimin, A. Kotsopoulos, J. L. Duarte, and M. A. M. Hendrix. "Transformer-Coupled Multiport ZVS Bidirectional Dc-Dc Converter with Wide Input Range." IEEE Transactions on Power Electronics, 23 no. 2, 771-781, 2008.

[20] J. E. Bosso, G. G. Oggier, and G. O. Garcia, "Isolated Buck/Boost Bidirectional DC-Three Phase Topology," IEEE Latin America Transactions, vol. 14, pp. 2669-2674, 2016.

[21] N. Mohan, T. M. Undeland, W. P. Robbins. "Power Electronics. Converters, Applications and design”. John Wiley \& Sons. 2003.

[22] Robert W. Erickson, Dragan Maksimovic. "Fundamentals of Power Electronics". Second Edition. 2001.

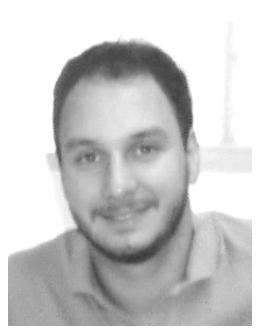

Jonathan Emmanuel Bosso (S'12-M'17) was born in Villa Mercedes, Argentina, in 1986. He received the Electronics Engineering degree from the Universidad Nacional de San Luis (UNSL), Argentina, in 2012 and the Doctor degree in engineering sciences from the Universidad Nacional de Rio Cuarto (UNRC), Argentina, in 2017. Since 2007, he is a member of Laboratorio de Control Automático, UNSL, and from 2012, he joined the Grupo de Electrónica Aplicada,
UNRC. $\mathrm{He}$ is also with the Consejo Nacional de Investigaciones Cientificas y Técnicas (CONICET), Buenos Aires, Argentina. His research interests are in power electronics, solid-state transformers, and renewable energy conversion.

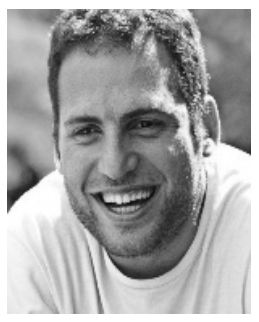

Marcelo Llomplat was born in San Miguel de Tucuman, Argentina, in 1984. In 2013 received the Electronics Engineering degree from Universidad Tecnológica Nacional, Facultad Regional Tucuman. From 2016, he joined the Grupo de Electrónica Aplicada, Universidad Nacional de Rio Cuarto (UNRC), Argentina. He is currently $\mathrm{PhD}$ student at the UNRC. He is also with the Consejo Nacional de Investigaciones Cientificas y Técnicas (CONICET), Argentina. His research interests are in power electronics, solid-state transformers, and renewable energy conversion.

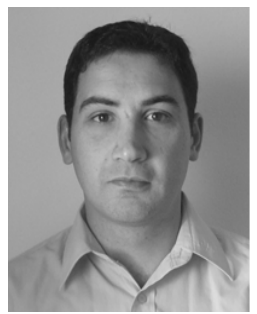

Germán Gustavo Oggier (M'10) was born in Córdoba, Argentina. He received the Electr. Eng. degree and the M.Sc. degree in electrical engineering from the Universidad Nacional de Rio Cuarto, Rio Cuarto, Argentina, in 2003 and 2006, respectively, and the Doctor degree in control systems from the Universidad Nacional del Sur, Buenos Aires, Argentina, in 2009. He is currently a Lecturer in the Grupo de Electronica Aplicada, Universidad Nacional de Rio Cuarto. $\mathrm{He}$ is also a Researcher with the Consejo Nacional de Investigaciones Científicas y Técnicas (CONICET), Buenos Aires, Argentina. His current research interests include power electronics, electric vehicles, and renewable energy conversion.

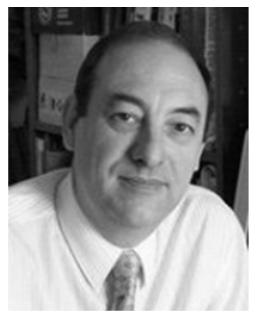

Guillermo Oscar Garcia (M'86-S'90 M'95-SM'01) was born in Río Cuarto, Argentina, in 1954. He received the electrical and electronics engineering degree from the Universidad Nacional de Córdoba, Córdoba, Argentina, in 1981 and the M.Sc. and Dr. degrees in electrical engineering from the Universidade Federal do Rio de Janeiro, Rio de Janeiro, Brazil, in 1990 and 1994, respectively. Since 1994, he has been with the Universidad Nacional de Río Cuarto, Río Cuarto, where he is currently the Director of the Grupo de Electrónica Aplicada. He is also with the Consejo Nacional de Investigaciones Científicas y Técnicas, Buenos Aires, Argentina. His research interests include power electronics, electric vehicles, and renewable energy conversion. 